\title{
Primacy of dimensions in vibrotactile perception: An evaluation of early holistic models
}

\author{
ROBERT D. MELARA and DUNCAN J. A. DAY \\ Purdue University, West Lafayette, Indiana
}

\begin{abstract}
Early holistic models of perception presume that stimuli composed of interacting dimensions can be experienced initially as undifferentiated. This view, formalized through recourse to a Euclidean geometry of perceptual space, predicts that the orientation of axes used to create stimulus sets is unimportant to performance in speeded classification. We tested this idea by using the interacting vibrotactile dimensions of pitch and loudness. Despite perceivers' relatively poor experience with these dimensions, we showed that the orientation corresponding to pitch and loudness was unique in vibrotactile perceptual space; subjects classified stimuli more efficiently at this orientation than at other orientations. Certain holistic models also claim that when stimulus differences are small, perceivers can recognize change without distinguishing the kind of change. We tested this idea by using a signal detection analysis of unspeeded same-different decisions. We found that subjects' ability to notice the kind of change equaled their ability to notice the change alone. In view of these results, which indicate that pitch and loudness are primary in vibrotactile perception, we detail a new conception of dimensional interaction.
\end{abstract}

What is the taproot of perceptual experience? Intuitively, it may seem that, at their core, percepts are experienced as if they are undifferentiated. A quick glance, for example, can provide us with a new experience, but one having a seemingly indeterminate nature. Accordingly, many researchers in cognitive psychology subscribe to the view that human beings' initial experiences are experiences of wholes that must then be analyzed if one wishes to recover their perceptual constituents. This general idea has held appeal in perceptual psychology at least since the seminal contributions of the Gestalt psychologists (e.g., Köhler, 1940; Wertheimer, 1S 15 ). In recent years, the idea has resumed its popularity in the form of early holistic models of perception (Garner, 1974b; Kemler, 1983a, 1983b; Lockhead, 1972, 1979; Shepp, 1989; Smith \& Kemler, 1977, 1978). All models in this class assume that our most basic perceptions are, or can be, perceptions of undifferentiated wholes or "blobs." Perceptual systems can then operate on (i.e., analyze) these wholes, but only with effort, to experience values on the individual dimensions that constitute the wholes.

In the present article, we evaluate early holistic models by using stimuli that represent seemingly prototypic ex-

The research reported in this article was supported by National Institutes of Health Grant NS28617 to Robert Melara. Portions of the report were presented at the 32nd Annual Meeting of the Psychonomic Society, which was held in San Francisco in November 1991. We appreciate the comments and suggestions of Edward Carterette, Wendell Garner, and an anonymous reviewer on an earlier version of this manuscript. Thanks go to Patricia Davies of Herrick Laboratories, Lisa Huffman, Michelle Anderson, and Cynthia Aguilar for their help with data collection and analyses. Address all correspondence to Robert D. Melara, Department of Psychological Sciences, Purdue University, West Lafayette, IN 47907. amples of early holistic processing-namely, vibrotactile stimuli varying in frequency and intensity. Most perceivers have little systematic experience with dimensions of vibrotaction. Certainly, our typical vibrotactile experiences do not nearly provide the opportunities to distinguish and identify changes in frequency and intensity as occur in, say, sound perception. Nonetheless, human beings do perceive changes in vibrotaction, although not as acutely as they perceive changes in vision or audition (see, e.g., Békésy, 1959; Khanna \& Sherrick, 1981; Rothenberg, Verrillo, Zahorian, Brachman, \& Bolanowski, 1977). One might expect, therefore, that perceivers would recognize that a vibrotactile change has occurred well before they can identify the nature of that change (e.g., that pitch has changed). The notion of perceiving undifferentiated change is exactly that espoused by early holistic models. Thus, consideration of vibrotactile processing provides a unique opportunity to test this entire class of models.

\section{Dimensional Interaction}

Several versions of the early holistic view are possible. In one, which is the focus of this article, perceptual systems integrate only certain kinds of dimensions, so that only these dimensions lead to holistic experiences. Such dimensions are called interacting, because the two or more sources of information that they represent are not treated independently by perceptual and/or other cognitive systems. The auditory dimensions of pitch and loudness exemplify a pair of interacting dimensions (Wood, 1975). In this article, we show that the vibrotactile dimensions of pitch and loudness also interact (see also Taylor, 1977).

Interacting dimensions are any dimensions that lead to Garner interference in speeded classification-when classifying values along one dimension (e.g., loudness), sub- 
jects suffer interference in performance (speed and accuracy) when the other dimension (e.g., pitch) is varied orthogonally from trial to trial (Garner, 1974b; Garner \& Felfoldy, 1970; Pomerantz, 1983, 1986). Technically, Garner interference is the difference in performance between a filtering task, in which the relevant and irrelevant dimensions are varied orthogonally (ordinarily, with two values on each dimension), and performance in a baseline task, in which the irrelevant dimension is held constant at one of its two values. Interacting dimensions are distinguished from separable dimensions, which, according to most accounts, do not yield undifferentiated stimulus wholes (but see Smith \& Kemler Nelson, 1984). Separable dimensions lead to no Garner interference in speeded classification, a result that defines separable dimensions operationally. One example is the pair of visual dimensions shape and quantity (Fitts \& Biederman, 1965; see also Egeth, 1967; Imai \& Garner, 1965). Apparently, these sources of information are treated independently by whatever systems the classification task activates.

A raison d'être of early holistic models is to explain why Garner interference is observed in the classification of some pairs of dimensions (interacting) but not others (separable). According to these models, Garner interference occurs when the number of wholes to be classified in filtering exceeds the number of wholes at baseline. With interacting dimensions, the dimensions themselves are not distinguished, meaning that each stimulus is experienced as a whole. Garner interference results because the filtering task contains four wholes (i.e., the four stimuli in the set), which must be mapped onto only two responses. The baseline task contains only two wholes, making it the easier task. Garner interference is not found between separable dimensions, because, according to early holistic models, perceivers experience each value on each dimension as a whole, yielding only two wholes on each dimension (e.g., high and low frequency) in both filtering and baseline classification. In the present article, we evaluate this explanation in the sphere of vibrotactile perception.

To recapitulate, early holistic models usually distinguish between stimuli composed of interacting dimensions, which are experienced initially as undifferentiated wholes, and stimuli composed of separable dimensions, whose constituents are recognized immediately in perception. Holistic processing of interacting dimensions may be followed by analytic processing to identify the constituents, but such analysis is presumed to require some effort. Thus, to distinguish interacting from separable dimensions, theorists have sought conditions that place subjects under speed stress to exclude the opportunity to perform extensive analysis of wholes (see Smith \& Kemler Nelson, 1984; Ward, 1983). Finally, holistic processing is presumed to underlie Garner interference in speeded classification.

\section{The Geometry of Early Holistic Models}

Early holistic models are specified typically through geometric formalisms (see Foard \& Kemler Nelson, 1984; Garner, 1974b; Lockhead, 1979). Stimuli created from interacting dimensions are represented as unanalyzed points in a psychological space. A Euclidean distance function is thought to organize this space:

$$
d_{i j}=\sqrt{\sum_{k=1}^{N}\left|x_{i k}-x_{j k}\right|^{2}},
$$

where $d_{i j}$ is the Euclidean distance between two stimuli, $i$ and $j, x_{i k}$ is the value of stimulus $i$ on dimension $k$, and $N$ is the number of dimensions of difference. Accordingly, distance relations are invariant with respect to the orientation of perpendicular reference axes. Put simply, holistic models assume that, in a psychological space representing stimuli composed of interacting dimensions, no reference axes are unique.

Most holistic models assume that specific reference axes are available to the perceiver as a consequence of cognitive effort through a subsequent analytic stage of processing (see, e.g., Garner, 1974b; Smith \& Kemler, 1978). In geometric terms, these models state that cognitive effort transforms a Euclidean map into a "city-block" map. A city-block function computes distance relations according to an additive rule; the distance between two points is the sum of the differences along each of a unique set of axes in the space:

$$
d_{i j}=\sum_{k=1}^{N}\left|x_{i k}-x_{j k}\right|
$$

Thus, the psychological distance between two sounds that differ in frequency and intensity would be the sum of the sounds' scaled differences in pitch and in loudness.

Early holistic models also assume that under speed stress, which can preclude expenditure of cognitive effort, perceivers rely on the Euclidean map (Garner, 1974b; Lockhead, 1972). Moreover, according to some holistic models, subjects perform optimally in identification tasks, in the sense that they are sensitive to what information is needed to meet the demands of the task (Foard \& Kemler Nelson, 1984). These ideas lead to specific predictions. Imagine four points that form a square in a Euclidean space, as in panel A of Figure 1. Imagine now that this same square is repositioned in the space, according to reference axes oriented $22.5^{\circ}$ or $45^{\circ}$ from the reference axes of panel A. These transformations are shown in panel B. Note that, because the space is Euclidean, the distance relations among points in the square do not change whether the axes are oriented at $0^{\circ}, 22.5^{\circ}$, or $45^{\circ}$.

One can conceive of this Euclidean map as the output of holistic processing, with each point corresponding to a stimulus representation in a psychological space, and each set of four points being a stimulus set. From the point of view of all early holistic models, the psychological distance among stimuli in the sets does not vary with changes in orientation of axes. Thus, under conditions that emphasize holistic processing (e.g., speed stress), performance in classifying stimuli within each set should be invariant across whatever axes are used to construct the stimulus set.

Of course, it is possible that speed stress does not prevent analytic processing completely. We then can ask, Is it optimal for subjects to perform speeded classification 
A.

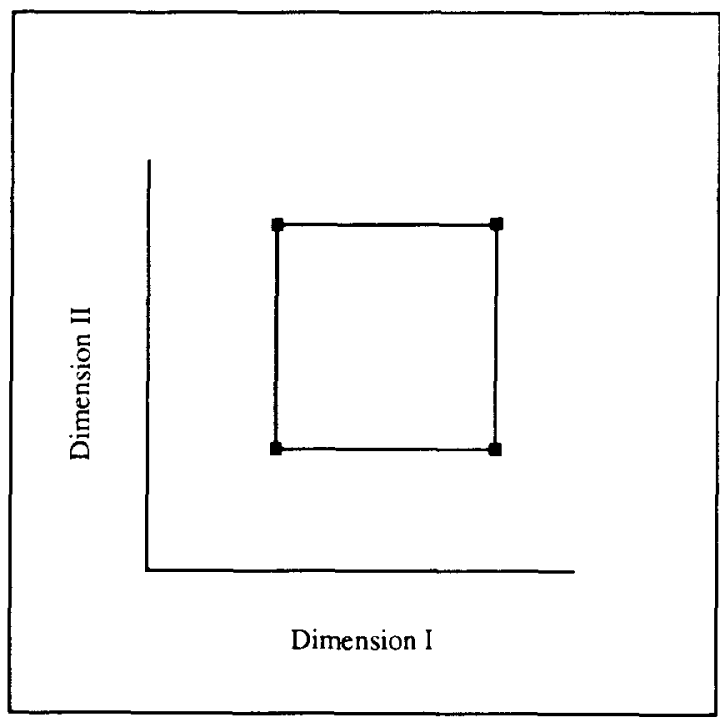

B.

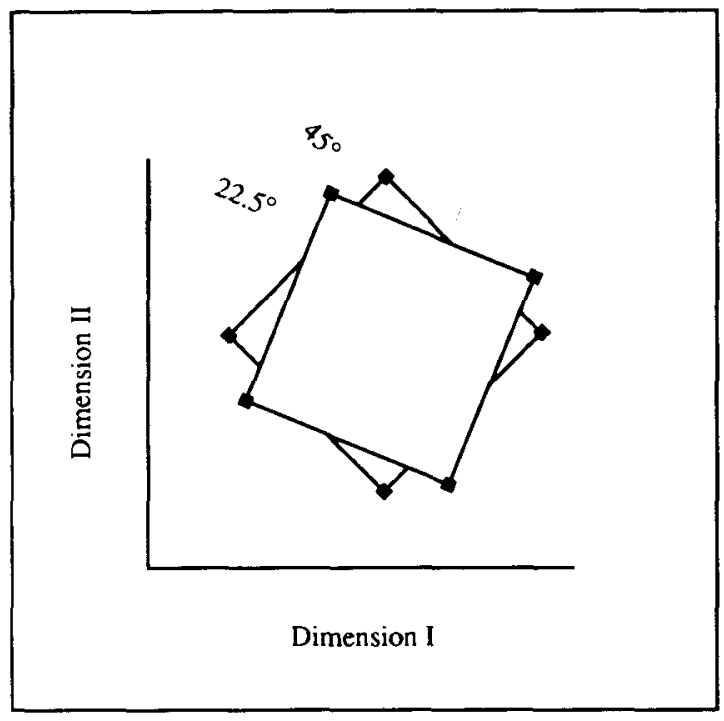

Figure 1. (A) Graphic representation of four stimuli differing equally along each of two dimensions in perceptual space. (B) Transformation of the original stimulus set by $22.5^{\circ}$ and $45^{\circ}$.

by first converting Euclidean distance into city-block distance? The answer is no, because distance relations in cityblock space always work against classification for sets constructed according to any reference axes other than those unique to the space. To see this, consider in Figure 2 the distance relations among three stimuli, A, B, and $C$, members of a square set created according to axes oriented $45^{\circ}$ relative to vertical-horizontal. Suppose the task was to place A and B into one category and C into another category. In city-block space, the task is uncomplicated, given that the $45^{\circ}$ axes are the unique axes. Stimuli $A$ and $B$, which receive the same response, project to the same point on the $45^{\circ}$ axes; Stimulus $C$, which receives a different response, projects to a different point. These projections are depicted by dotted lines in Figure 2. However, if in this example any but the $45^{\circ}$ orientation is unique, the response mapping does not match the stimulus projections. In Figure 2, we use dashed lines to represent projections in city-block space when the unique reference axes are the vertical-horizontal axes. Note how difficult the classification task becomes: $\mathrm{A}$ and $\mathrm{B}$, from the same category, project to different points on the abscissa, but $\mathrm{A}$ and $\mathrm{C}$, from different categories, project to the same point. By contrast, in Euclidean space, the distance relations are the same no matter how the square is oriented in the space. Indeed, the Euclidean distance between $A$ and $B$ is always smaller (i.e., less confusable) than the corresponding city-block distance, except at the unique orientation in city-block space. And even at this unique orientation, $A-B$ city-block distance merely equals A-B Euclidean distance.

Thus, except in special cases (i.e., when the perceptual space and the classification space are defined by the same axes), city-block distance is inferior to Euclidean distance for efficiency of classification. As shown in Figure 3, the Euclidean map leads to constant performance across orientation of axes (panel A), whereas the cityblock map leads to worse performance at orientations other than that unique to the space (panel B). According to early holistic models, therefore, subjects would have no use for a city-block map in speeded classification of interacting dimensions, because a Euclidean map is readily available. Conversely, if evidence that resembled the pattern shown in panel B was obtained, we might then conclude that subjects did not have special access to a Euclidean map. $\mathrm{Ob}$ taining this pattern would thus serve as evidence against the class of early holistic models.

\section{Evidence with Auditory Dimensions}

Melara and Marks (1990a) tested predictions of early holistic models by using a technique that required subjects to perform speeded classification of stimulus sets that were created according to three orientations of axes in auditory perceptual space: $0^{\circ}, 22.5^{\circ}$, and $45^{\circ}$ (cf. Foard \& Kemler Nelson, 1984; Grau \& Kemler Nelson, 1988). These stimulus sets are depicted in panel $A$ of Figure 4. Each set formed a square in psychological space, meaning that the stimuli within each set were perceptually equidistant along each dimension. At each orientation, subjects performed a task of selective attention-that is, the filtering taskand a task of divided attention, called a condensation task. The filtering task required subjects to classify values along one dimension (e.g., pitch) and to ignore variation along the other dimension (e.g., loudness). The condensation task required subjects to classify conjunctions of values. For example, subjects would make one response to highpitched/loud sounds or low-pitched/soft sounds, and a different response to high-pitched/soft sounds or low-pitched/ loud sounds.

At each orientation, performance in filtering and condensation was compared with baseline to form measures 


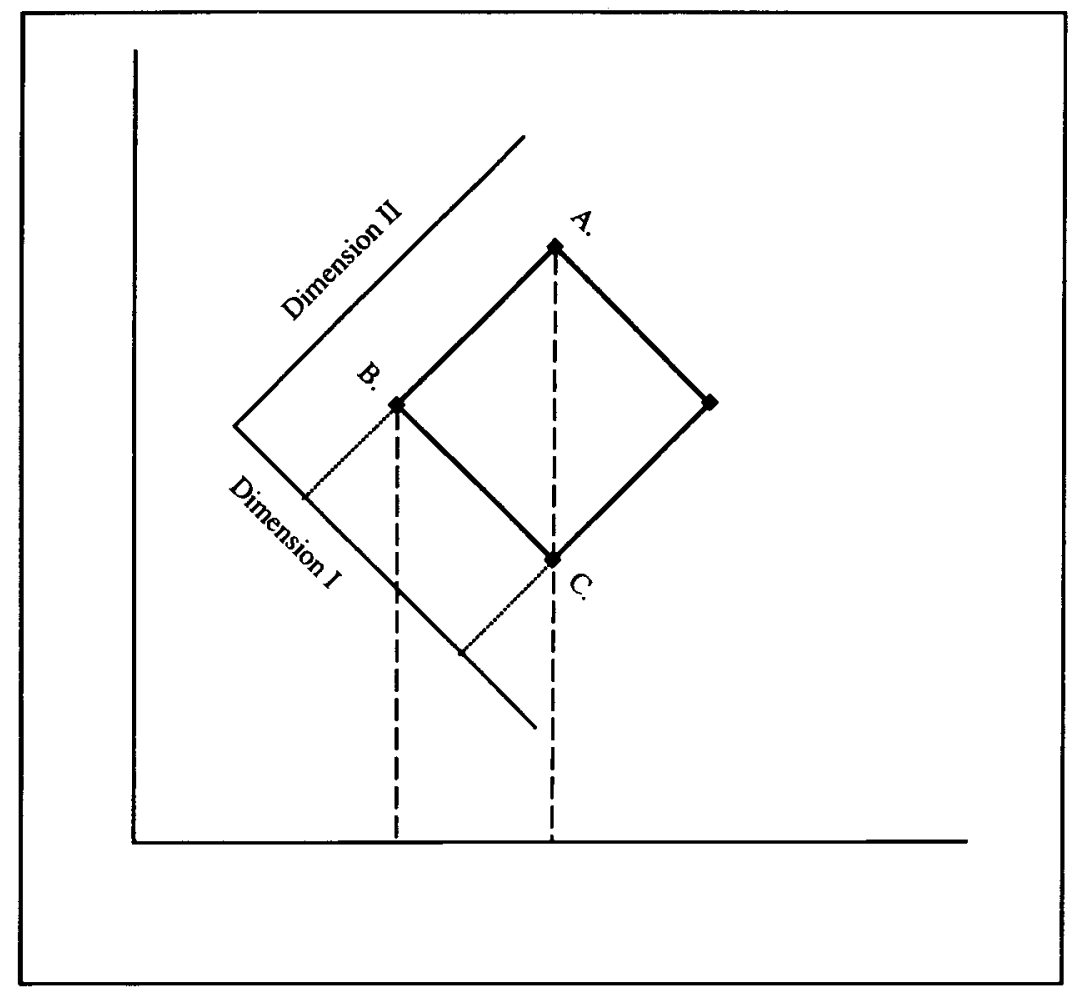

Figure 2. Graphic representation of the distance relations among three stimuli, A, B, and $C$, members of a square set in perceptual space, created according to reference axes oriented $45^{\circ}$ from vertical-horizontal, and with $A$ and B members of a single category. Category relations in city-block space are projected onto one of the classification axes when these axes are either identical to the reference axes (dotted lines) or vertical-horizontal and thus oriented $45^{\circ}$ relative to the reference axes (dashed lines).

of selective attention and divided attention, respectively. Early holistic models predict that performance in these attention tasks should not be affected by orientation of axes. As one can see in panel B of Figure 4, however, the results showed that as axes changed in orientation from $0^{\circ}$ to $45^{\circ}$, performance at selective attention deteriorated and performance at divided attention improved.

At present, we concentrate only on results with selective attention. Note that the pattern obtained follows that expected from a city-block map (see panel B of Figure 3) and is unlike that expected from a Euclidean map (panel A of Figure 3). The results thus run counter to the early holistic account. Moreover, as Melara and Marks (1990a) suggested, these data are consistent with models of primary dimensions. According to these models, which will be considered more fully in the General Discussion section, perceptual systems necessarily code stimuli along the set of psychologically important continua that constitute the stimuli. These continua, called primary dimensions, are thought to emerge from fundamental constraints of processing, what we refer to as mandatory processes (see Garner, 1974a; Melara, 1990). A model of primary dimensions implies that interacting and separable dimensions cannot be distinguished according to whether or not subjects have access to unique dimensions in the space, because both kinds of dimensions enjoy such access. One is thus forced to explain Garner interference without invoking holistic processing, because, as Melara and Marks (1990a) have shown, significant Garner interference obtains in the face of evidence against holistic processing.

At least two alternative accounts of Melara and Marks's (1990a) findings are possible, though, both of which involve modification of early holistic models. First, perhaps holistic processing and analytic processing are both mandatory, with holistic processing causing Garner interference at the $0^{\circ}$ orientation, and analytic processing causing the worsened performance at the other orientations. The difficulty with this modification, however, is that only analytic processing (i.e., primary dimensions) is dictated by the data. The only role of holistic processing is to account for a constant-that is, the Garner interference at the $0^{\circ}$ orientation. It is certainly unparsimonious to invoke an entire mechanism (i.e., a holistic processor) to deal with a constant term. In its place, one could claim that Garner interference is due to the time required to establish the unique coordinate axes in city-block space. This time presumably varies with the dimensions being paired; thus, it seems plausible that time is extremely brief with sepa- 
A.

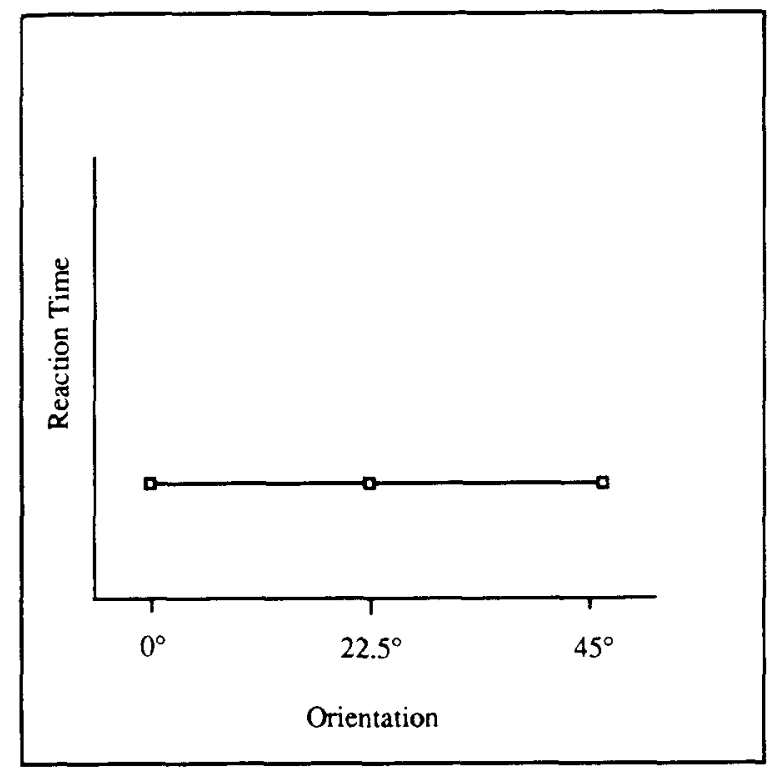

B.

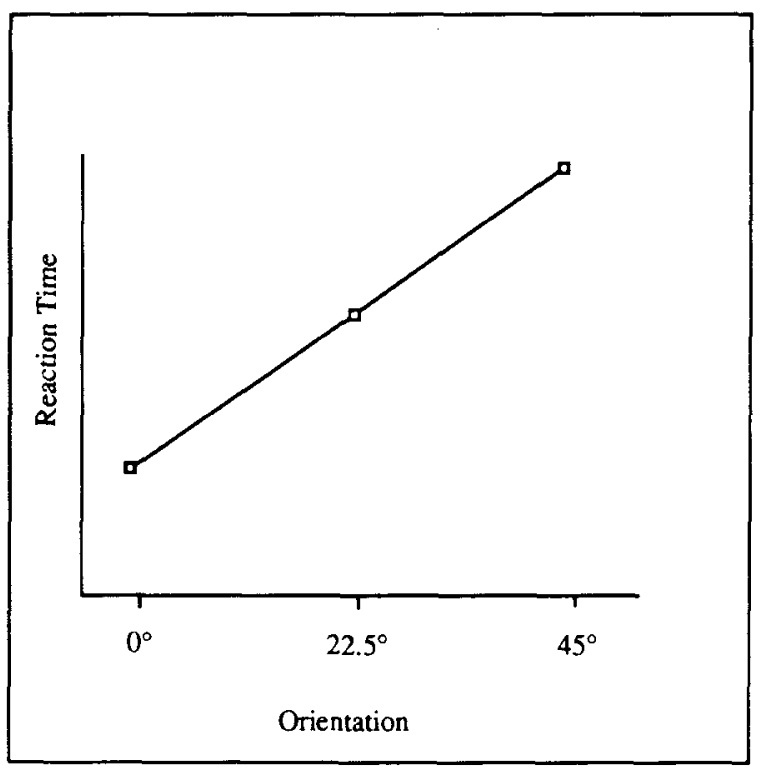

Figure 3. Graphic depiction of predicted classification performance based on a Euclidean (holistic) map (panel $A$ ) and a city-block (primary dimensions) map (panel B).

rable dimensions, causing no Garner interference, and relatively long with interacting dimensions, causing substantial Garner interference. (These ideas are addressed more fully in the General Discussion section.) However the analytic mechanism may be characterized, Melara and Marks's data argue against the requirement in any model of a mandatory holistic stage, at least between auditory dimensions.

A second, more viable account states that dimensions of sound are special. In this view, auditory dimensions have some probability of invoking holistic processing and some probability of invoking analytic processing (see Grau $\&$ Kemler Nelson, 1988). Auditory dimensions interact and so initially may be processed holistically. However, we can identify the constituents of sound more easily than the constituents of stimuli formed from other interacting dimensions, presumably because of our extensive experience with sound dimensions. Thus, these dimensions may also lead to analytic processing in speeded classification, or to some combination thereof. A combination of holistic processing and analytic processing could explain the pattern obtained by Melara and Marks (1990a).

This second alternative account differs from the first, because it regards perceptual processing as highly malleable through experience. In other words, the second account considers processing to be optionally holistic (or optionally analytic), a name we shall use henceforth in referring to this alternative (see Garner, 1974a). The optional holistic model suggests that one can predict the probability that interacting dimensions are processed analytically through knowledge of perceivers' prior experience with the dimensions.

In this regard, the vibrotactile dimensions of pitch and loudness represent a good test case of the optional holistic account, because perceivers typically have little organized experience with these dimensions. It is unlikely, for example, that the average perceiver has ever had cause to distinguish consciously a change in vibrotactile pitch from a change in vibrotactile loudness. Hence, according to the optional holistic account, one would expect little, if any, evidence that perceivers accord special status to specific dimensions of vibrotactile stimuli, at least relative to auditory stimuli. In the present study, we evaluated this hypothesis by subjecting vibrotactile dimensions to several tests of holistic processing. The results will support the claim that one does not need holistic processing, of either the mandatory or the optional variety, in order to account for interaction between vibrotactile dimensions.

\section{EXPERIMENT 1}

It is first necessary to confirm an interactive relation between dimensions of vibrotaction. Garner and his colleagues (e.g., Garner, 1974b; Garner \& Felfoldy, 1970) developed an experimental procedure for diagnosing instances of dimensional interaction. The method consists of speeded classification, and the complete paradigm contains three kinds of tasks: (1) baseline tasks, in which the irrelevant dimension is held constant; (2) filtering tasks, in which the relevant dimension and the irrelevant dimension are varied orthogonally; and (3) correlated tasks, in which values on the irrelevant dimension are paired redundantly with values on the relevant dimension. When interacting dimensions are classified, the latter task frequently leads to performance that is superior to baseline, an effect known as redundancy gain.

The speeded classification procedure thus provides two indices of interaction between dimensions: (1) Garner interference when the filtering tasks are performed; and 

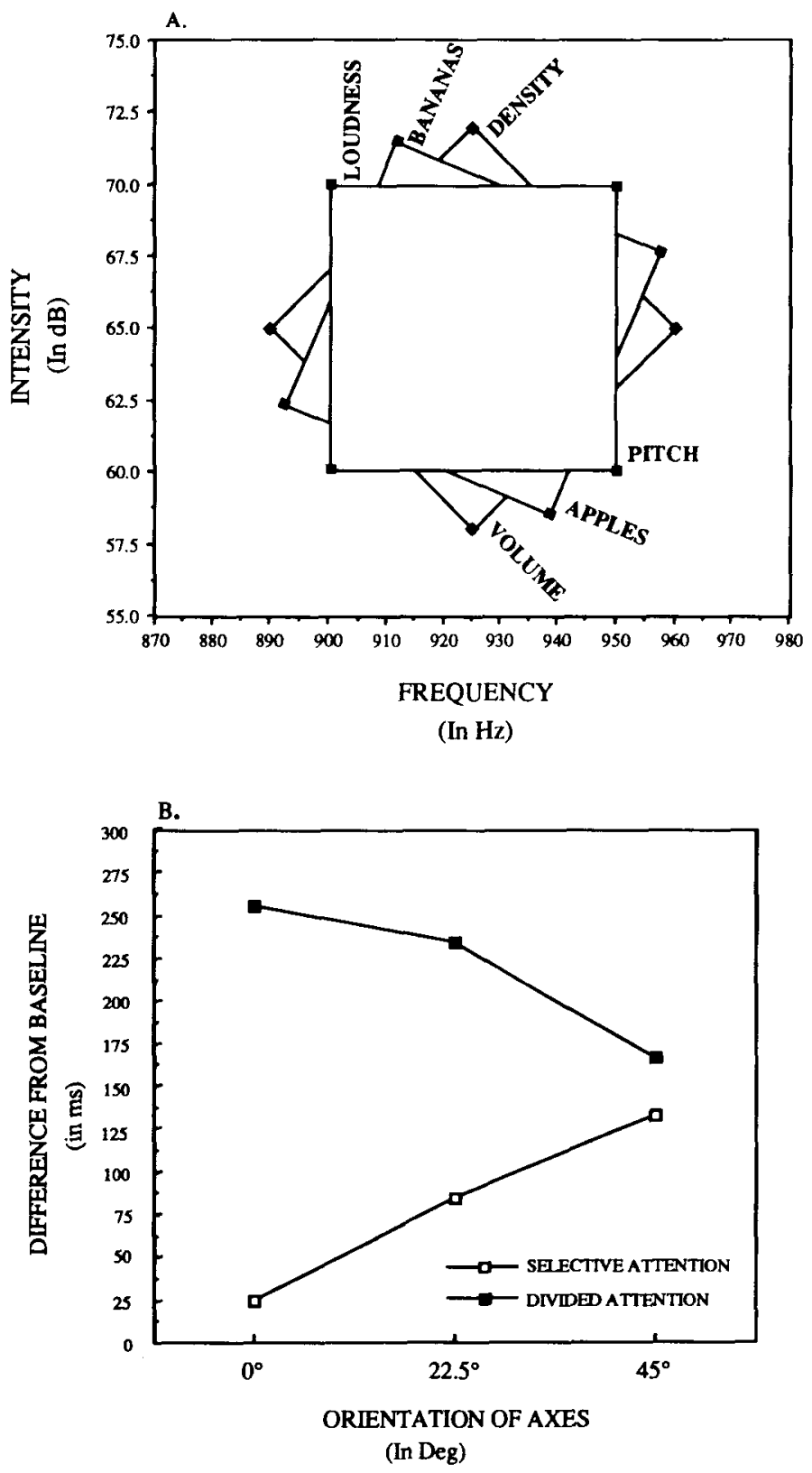

Figure 4. (A) Stimulus set used by Melara and Marks (1990a; Experiments 1 and 2 ), created from three orientations of frequency-intensity axes: $0^{\circ}$ (pitch-loudness), 22.5 ("apples"-bananas"), and $45^{\circ}$ (volume-density). (B) Mean difference from baseline performance (in milliseconds) in filtering tasks (selective attention) and condensation tasks (divided attention) at each of three orientations of frequency-intensity axes in Melara and Marks's (1990a) Experiment 2. [From "Perceptual primacy of dimensions: Support for a model of dimensional interaction," by R. D. Melara \& L. E. Marks, 1990, Journal of Experimental Psychology: Human Perception \& Performance, 16, 402 and 406. Copyright 1990 by the American Psychological Association, Inc. Panel $A$ is adapted, panel $B$ reprinted, by permission of the publisher.] 
(2) redundancy gain when the correlated tasks are performed. In Experiment 1, we ask whether or not the vibrotactile dimensions of pitch and loudness interact in speeded classification (see also Taylor, 1977).

\section{Method}

Subjects. Twenty right-handed undergraduates at Purdue University (12 men, 8 women) participated in partial fulfillment of a course requirement. None had extensive experience with vibrotactile stimuli, at least as measured by self-report.

Stimuli and Apparatus. Sine-wave signals were generated by an Amiga 500 microcomputer, amplified by a Hewlett-Packard HP465A amplifier, and transduced through a Brüel and Kjær 4810 minishaker. The vibrating piston of the minishaker had a circular surface area of $7.85 \mathrm{~cm}^{2}$, and a surface thickness of $7 \mathrm{~mm}$, sufficient to mitigate any spatial effects (i.e., cues) of frequency change. The contactor was covered with no. 60 sandpaper to help secure a constant finger position. The minishaker was housed in a foaminsulated wooden box to attenuate auditory vibration. To further eliminate sound information from the minishaker, subjects listened to white noise for the duration of the experiment. The noise was supplied by a JIL 872 white noise generator and was heard over both Nova-40 headphones and from an external speaker located directly above the minishaker. The intensity of each source of noise was $72 \mathrm{~dB}(\mathrm{~A})$. Subjects felt vibrations by placing the left index finger on the vibrating surface. They were instructed to maintain a constant amount of pressure on the surface. Each subject's left arm rested on a cushioned arm support. Responses were made with the index and middle finger of the right hand on a Naksha dual-key mouse. Instructions and feedback were provided on a Commodore 1084A color monitor.

The classification set contained four stimuli, two values of frequency at two levels of intensity. Extensive psychophysical testing was performed to match pairs of frequencies at a given intensity level in loudness and to match pairs of intensities at a given frequency level in pitch (see Békésy, 1967, p. 167). The resulting four stimuli were $210 \mathrm{~Hz}$ at $93 \mathrm{~dB} ; 210 \mathrm{~Hz}$ at $101 \mathrm{~dB} ; 315 \mathrm{~Hz}$ at $92 \mathrm{~dB}$; and $315 \mathrm{~Hz}$ at $101 \mathrm{~dB}$. The intensities were calibrated with a PCB 309A accelerometer, amplified by a PCB 482 A04 preamplifier, and displayed on a Heath 4251 oscilloscope. The intensities are expressed in decibels, using $10^{-11} \mathrm{~m}$ as the reference displacement amplitude (see Beranek, 1988). The frequencies were calibrated with a Heath IM 2410 frequency counter and the Heath oscilloscope.

The stimuli were used to create five classification tasks for each of two classification dimensions, pitch and loudness. On each trial of the two baseline tasks, the classification dimension (e.g., pitch) could take on either of its two values (high or low pitch), while the irrelevant dimension (e.g., loudness) was held constant at one of its two values (e.g., soft): On each trial of the filtering task, both the relevant and irrelevant dimensions could take on either of their two values. On each trial of the correlated tasks, the value on the relevant dimension was matched to a corresponding value on the irrelevant dimension. In the positively correlated task, for example, high pitch was paired with loud and low pitch was paired with soft. Each stimulus in a trial lasted $1,100 \mathrm{msec}$. Each task contained 48 trials, maintaining equal numbers of two (baseline, correlated) or four (filtering) stimuli. Within each task, stimuli were presented in random order.

Procedure. Subjects were tested individually in an IAC 1201 sound-attenuating chamber. The subjects performed all five tasks for one dimension as a set, followed by all tasks for the other dimension. Order of task within a set was specified by Latin square; order of dimension of classification was counterbalanced across subjects. Before each set, the subjects performed 96 trials of practice in the baseline task. These data were discarded.

The subjects were instructed to attend to the relevant dimension and to ignore variation along the irrelevant dimension. They were told to respond fast but accurately. Their responses were recorded in milliseconds using a hardware timer (Wright, 1986). The subjects received error feedback on each trial. Trials whose latency exceeded 1,200 msec were repeated at a later point in the task. Tasks whose accuracy fell below $85 \%$ were repeated immediately. After each task, the subjects were informed of their overall speed and accuracy. Each task was performed twice consecutively; analyses were performed on the average of the two runs. The entire experiment lasted approximately $50 \mathrm{~min}$.

\section{Results and Discussion}

Mean reaction times (RTs) for each condition of each classification dimension appear in Figure 5. The overall error rate was .03 . The high accuracy of our subjects indicates that performance did not suffer as a result of their continuous involvement with the small set of (four) vibrotactile stimuli. Speed correlated .90 with accuracy, computed according to mean performance in each of the 10 tasks. Analyses of variance (ANOVAs) were performed on both RTs and error proportions. As is evident in Figure 5, the subjects classified pitches $(533 \mathrm{msec}) 28 \mathrm{msec}$ slower than they classified loudnesses $(505 \mathrm{msec})[F(1,19)$

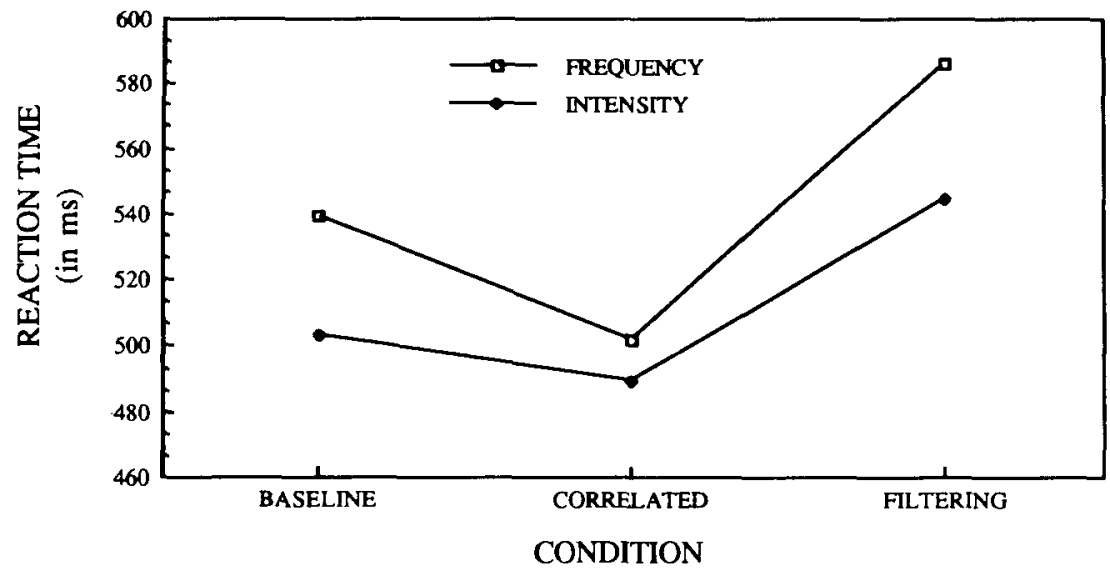

Figure 5. Average reaction time performance in milliseconds for classifying vibrotactile pitch and loudness in baseline, correlated, and filtering tasks (Experiment 1). 
$\left.=8.98, M S_{\mathrm{e}}=4,282.88, p<.01\right]$, indicating unequal psychological distance along dimensions. As we shall see, despite this mismatch, a consistent pattern emerged, although the less discriminable dimension (pitch) was affected more by the more discriminable dimension (loudness) than vice versa (see Figure 5). There was also an interaction between dimension (pitch, loudness) and task condition (five levels) $\left[F(4,76)=5.44, M S_{\mathrm{c}}=1,054.74\right.$, $p<.001]$. This was due to the fact that subjects showed no redundancy gain when judging loudness in the positively correlated task. Otherwise, as can be seen in Figure 5 , the pattern of performance across tasks was similar between the two dimensions. Moreover, this interaction did not obtain in analysis of error proportions $[F(4,76)$ $=.82, M S_{\mathrm{e}}=.02$, n.s.]. Hence, our principal analyses reflect performance averaged across dimensions.

We found a main effect of condition $[F(4,76)=20.60$, $\left.M S_{\mathrm{e}}=918.85, p<.001\right]$. To evaluate the nature of this effect, a series of planned contrasts was performed in which averaged baseline performance was compared with performance in the filtering tasks and in the correlated tasks, using pooled within-subject effects to derive the error terms (Winer, 1971). The main results can be summarized easily. The subjects suffered significant Garner interference. The average speed in filtering $(565 \mathrm{msec})$ was $44 \mathrm{msec}$ slower than the average speed at baseline $(521 \mathrm{msec})[F(1,76)=28.97, p<.001]$. In addition, errors in filtering (.05) were greater than errors at baseline $(.03)\left[F(1,76)=35.13, M S_{\mathrm{c}}=.01, p<.001\right]$. The subjects also experienced a reliable redundancy gain, at least in RT. The average speed when dimensions were correlated $(495 \mathrm{msec}$ ) was $26 \mathrm{msec}$ faster than baseline $[F(1,76)$ $=14.21, p<.001]$. Redundancy gain was not observed in errors, however; both baseline and correlated error proportions averaged $.03[F(1,76)=.59$, n.s. $]$.

These results demonstrate clearly that the vibrotactile dimensions of pitch and loudness interact in speeded classification. Subjects cannot attend to one dimension without being affected, either positively or negatively, by variation on an irrelevant dimension. In the remaining experiments, we explored the nature of this interaction.

\section{EXPERIMENT 2}

Early holistic models attribute Garner interference to holistic processing. The optional holistic model, as described in the introduction, states that subjects can become more analytic as they gain experience with a perceptual dimension. The model thus implies that subjects should improve in their ability to overcome the detrimental effects of orthogonal variation with continued exposure to the task. We tested this idea in Experiment 2 by tracking Garner interference over the course of practice. Specifically, we compared baseline performance with filtering performance across 14 blocks of 96 trials.

Our goal is to gauge reliable diminutions in Garner interference as subjects become more proficient with vibrotactile classification. If the causes of Garner interference are optional to any degree, whether or not these causes lie in holistic processing, we would expect subjects to improve in their ability to attend selectively over the course of trials. Indeed, Garner interference between certain nonperceptual dimensions, such as Stroop dimensions, quickly disappears following brief periods of practice (Melara \& Marks, 1990b). Of course, because the window of change that we examine is finite-indeed, only a single experimental session-a failure to eliminate interference effects cannot be attributed with certainty to the role of mandatory processes. Nevertheless, such a finding would be instructive regarding the malleability of interactive processes.

\section{Method}

Subjects. Thirty-two subjects (18 men, 14 women) participated in partial fulfillment of a course requirement. None had participated in Experiment 1.

Stimuli, Apparatus, and Procedure. The stimuli and apparatus were identical to those in Experiment 1. Only the baseline and filtering conditions of pitch classification were tested. After baseline practice, subjects alternated between a baseline task and a filtering task for 14 blocks of trials. Order of alternation, baseline first or filtering first, was counterbalanced across subjects. In the baseline condition, the particular level of the irrelevant dimension alternated across blocks. The subjects were instructed to try to improve in their ability to ignore loudness while classifying pitch. Otherwise, the procedure was identical to that in Experiment 1. The experiment lasted approximately $1 \mathrm{~h}$.

\section{Results}

Average RTs to baseline and filtering conditions in each block of trials appear in Figure 6. An ANOVA of RTs was performed with block (14 levels) and condition ( 2 levels) as factors. There was a main effect of block $[F(13,403)=$ $\left.7.00, M S_{\mathrm{e}}=6,234.19, p<.001\right]$, because the subjects' speed at classifying improved from an average of $585 \mathrm{msec}$ in Block 1 to an average of $501 \mathrm{msec}$ by Block 11. There was also a main effect of condition $[F(1,31)=66.65$, $\left.M S_{\mathrm{e}}=8,603.18, p<.001\right]$, indicating that subjects experienced significant Garner interference. The average performance in filtering $(562 \mathrm{msec}$ ) was $50 \mathrm{msec}$ slower than the average performance at baseline $(512 \mathrm{msec})$. There was no interaction between block and condition $\left[F(13,403)=.43, M S_{e}=2,614.72\right.$, n.s. $]$. As one can see in Figure 6, Garner interference-the difference between baseline and filtering-remained almost constant over the course of blocks. A contrast was performed on difference scores, in which the average Garner interference in the first two blocks of trials was compared with that in the last two blocks of trials. The average interference was $57 \mathrm{msec}$ at both times $\left[F(1,403)=.20, M S_{\mathrm{e}}=\right.$ $7,148.24$, n.s.]. The subjects showed virtually no evidence of improvement in selective attention, despite the fact that they showed clear improvement in classification.

The pattern of errors was consistent with RTs. An ANOVA of error proportions revealed a significant effect of condition $\left[F(1,31)=56.66, M S_{\mathrm{e}}=.03, p<\right.$ $.001]$, with subjects committing more errors on filtering trials (.034) than on baseline trials (.026). Errors did not differ by block $\left[F(13,403)=.74, M S_{e}=.03\right.$, n.s. $]$, nor 


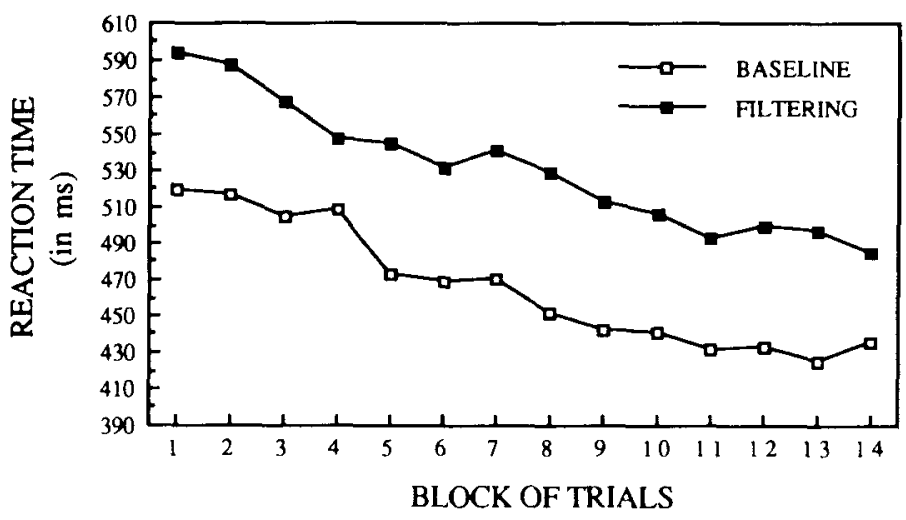

Figure 6. Average reaction time performance in milliseconds for classifying vibrotactile pitch while ignoring vibrotactile loudness in 14 blocks of 96 trials, alternating between a baseline task and a filtering task every 48 trials (Experiment 2).

was there a significant interaction between condition and block $\left[F(14,403)=1.60, M S_{e}=.03\right.$, n.s. $]$. The high overall level of accuracy, combined with the improvements in overall classification speed, suggest that prolonged exposure to a few vibrotactile stimuli caused no harm in classification performance.

\section{Discussion}

The results of Experiment 2 demonstrate that Garner interference between vibrotactile dimensions is not easily erased by practice. Despite 1,344 trials of classification experience, subjects showed no success in reducing interference effects. What conclusions can we draw from these results? First, they suggest that if continued practice at classifying vibrotactile dimensions does cause a change from holistic processing to analytic processing, such changes are not apparent within a single experimental session. This allows us to be relatively confident that our upcoming tests for holistic processing among naive subjects do not lead, in and of themselves, to a perceptual change toward analytic processing.

Second, the results indicate that vibrotactile dimensions are particularly apropos for testing early holistic models. The magnitude of Garner interference was enormous, as such effects go, and it showed no sign of diminution. These findings imply that an understanding of vibrotactile interactions will be informative about the nature of perceptual interaction in general. Although they do not rule out the optional holistic account, these results certainly constrain how quickly such optional mechanisms can be expected to operate. Indeed, the results are consistent with a model of mandatory interaction between vibrotactile dimensions, perhaps even mandatory holistic processing. A mandatory holistic mechanism, if it exists, would be limited in generality, however, because as indicated earlier, such an account is unsatisfactory for explaining auditory interaction. The remainder of this study evaluates whether interaction between vibrotactile dimensions is ex- plained best by this holistic mechanism or instead by postulating primary dimensions in perceptual space.

\section{EXPERIMENTS 3A AND 3B}

We now report measures of performance in selective attention and divided attention to stimuli created from various orientations of axes in vibrotactile perceptual space. The method is that developed by Melara and Marks (1990a), called selective/divided rotation, which is itself borrowed from ideas of Kemler Nelson and her colleagues (Foard \& Kemler Nelson, 1984; Smith \& Kemler, 1978). Subjects perform baseline, filtering, and condensation tasks at each of three orientations: $0^{\circ}, 22.5^{\circ}$, and $45^{\circ}$. Through pretesting, we determined that the stimulus set at each orientation formed a perceptual square in the space, at least among the subjects tested in these experiments. We asked whether orientation matters to speeded classification, or whether vibrotactile stimuli are processed holistically according to Euclidean spatial relations.

Experiments $3 \mathrm{~A}$ and $3 \mathrm{~B}$ differ in the range of vibrotactile frequencies tested. Psychophysical and physiological evidence has identified at least three different touch mechanoreceptor systems (see, e.g., Freeman \& Johnson, 1982; Gescheider, 1976; Gescheider, Frisina, \& Verrillo, 1979; Verrillo, 1963, 1966, 1985; see Sherrick \& Cholewiak, 1986, for a review). The Pacinian system, the focus of these experiments, is based on stimulation of Pacinian corpuscles deep in the epidermal layer. These cells have an optimal response sensitivity between 260 and $300 \mathrm{~Hz}$, but they are responsive to signals ranging from 40 to $800 \mathrm{~Hz}$. They project to the central nervous system directly-that is, as primary sensory afferents through the medial lemniscus. At lower frequencies, a slowly adapting system (SAl) and a rapidly adapting system (RA) operate in tandem on the basis of stimulation to Merkel endings and Meissner corpuscles, respectively. The SA1 and RA systems are particularly interesting because of their suspected 
roles in fine tactile discrimination of edge and shape information (see lggo, 1985).

In Experiment $3 \mathrm{~A}$, a frequency range particular to the Pacinian system was tested. Signals ranged between 118 and $337 \mathrm{~Hz}$, in this way largely restricting stimulation to Pacinian corpuscles. The frequency range was lowered in Experiment 3B, to between 58 and $207 \mathrm{~Hz}$. This region falls outside the optimal Pacinian response range, but triggers receptor activity in the non-Pacinian SAl and RA systems. By testing two different frequency ranges, which in turn activated different proportions of cell populations, we hoped to reach general conclusions about human perception of vibrotactile stimuli. As we shall see, the two frequency ranges led to a similar pattern of results.

\section{Method}

Subjects. Ten subjects ( 5 men, 5 women) from the Purdue community volunteered to participate in Experiments $3 A$ and $3 B$. All were right-handed.

Stimuli and Apparatus. The apparatus was identical to that in the earlier experiments. In each experiment, a set of four stimuli was created at each of three orientations in vibrotactile perceptual space: $0^{\circ}, 22.5^{\circ}$, and $45^{\circ}$. The $0^{\circ}$ orientation corresponded to the vibrotactile dimensions of pitch and loudness.

In Experiment $3 \mathrm{~A}$, the $0^{\circ}$ stimulus set was that used in Experiments 1 and 2: [a] $210 \mathrm{~Hz}$ at $93 \mathrm{~dB}$; [b] $210 \mathrm{~Hz}$ at $101 \mathrm{~dB}$; [c] $315 \mathrm{~Hz}$ at $92 \mathrm{~dB}$; and [d] $310 \mathrm{~Hz}$ at $101 \mathrm{~dB}$. These values were postmultiplied by an orthogonal transformation matrix that rotated axes in the space either $22.5^{\circ}$ or $45^{\circ}$. The resulting four stimuli at $22.5^{\circ}$ were [a] $194 \mathrm{~Hz}$ at $98 \mathrm{~dB}$; [b] $291 \mathrm{~Hz}$ at $92 \mathrm{~dB}$; [c] $331 \mathrm{~Hz}$ at $100 \mathrm{~dB}$; and [d] $234 \mathrm{~Hz}$ at $102 \mathrm{~dB}$. The stimulus set at the $45^{\circ}$ orientation consisted of: [a] $188 \mathrm{~Hz}$ at $99 \mathrm{~dB}$; [b] $263 \mathrm{~Hz}$ at $89 \mathrm{~dB}$; [c] $263 \mathrm{~Hz}$ at $101 \mathrm{~dB}$; and [d] $337 \mathrm{~Hz}$ at $96 \mathrm{~dB}$.

In Experiment $3 \mathrm{~B}$, the frequency range was lowered, creating the following $0^{\circ}$ stimulus set: [a] $80 \mathrm{~Hz}$ at $111 \mathrm{~dB}$; [b] $80 \mathrm{~Hz}$ at $118 \mathrm{~dB}$; [c] $185 \mathrm{~Hz}$ at $99 \mathrm{~dB}$; and [d] $185 \mathrm{~Hz}$ at $110 \mathrm{~dB}$. As before, psychophysical pretesting ensured that two frequencies at a given intensity level were equally loud, and that two intensities at a given frequency level were equal in pitch. The $0^{\circ}$ stimulus set was transformed to create sets at $22.5^{\circ}$ and $45^{\circ}$. The $22.5^{\circ}$ set comprised the following stimuli: [a] $64 \mathrm{~Hz}$ at $113 \mathrm{~dB}$; [b] $104 \mathrm{~Hz}$ at $107 \mathrm{~dB}$; [c] $161 \mathrm{~Hz}$ at $102 \mathrm{~dB}$; and [d] $201 \mathrm{~Hz}$ at $105 \mathrm{~dB}$. The $45^{\circ}$ set contained these four stimuli: [a] $58 \mathrm{~Hz}$ at $115 \mathrm{~dB}$; [b] $133 \mathrm{~Hz}$ at $103 \mathrm{~dB}$; [c] $133 \mathrm{~Hz}$ at $113 \mathrm{~dB}$; and [d] $207 \mathrm{~Hz}$ at $103 \mathrm{~dB}$.

From these sets, two baseline tasks and one filtering task were created for each dimension (i.e., two dimensions at each orientation), and one condensation task was created for each pair of dimensions. Using the lettering system outlined above, at each orientation the stimuli discriminated in four baseline tasks were (1) [a] versus [b]; (2) [a] versus [c]; (3) [b] versus [d]; and (4) [c] versus [d]. The stimuli classified in the two filtering tasks were: (1) [a] or [b] versus [c] or [d]; and (2) [a] or [c] versus [b] or [d]. These tasks correspond to the previous definitions for baseline and filtering tasks. In the condensation task, subjects placed stimuli with two high values or two low values on both dimensions in one category, and stimuli with a high value on one dimension and a low value on the other dimension in the other category: [a] or [d] versus [b] or [c].

Procedure. The subjects were tested individually in the IAC chamber. Each subject performed, at each orientation of axes, two baseline tasks (one per dimension), the two filtering tasks, and the condensation task. The particular baseline tasks tested were counterbalanced across subjects. The order of orientation set was counterbalanced; the subjects were permitted to rest briefly between sets. The order of tasks within an orientation set was determined ran- domly. The subjects were informed that each set was defined by a pair of dimensions and that their task was to classify values along one or both of these dimensions, depending on the task. Each subject performed each task twice consecutively, averaged for the purpose of analysis. Tasks performed at less than $85 \%$ accuracy were repeated immediately. Experiment $3 B$ was administered after Experiment $3 \mathrm{~A}$, separated by at least several weeks. In all other respects, the procedure duplicated that of Experiment 1. Each experiment lasted approximately $3 \mathrm{~h}$.

\section{Results}

Performance in selective attention and divided attention at each orientation, computed by subtracting RTs at baseline from RTs in filtering and condensation, respectively, appear in panel A (Experiment 3A) and panel B (Experiment 3B) of Figure 7. The overall error rate was .06 in Experiment 3A and .07 in Experiment 3B. Speed and accuracy correlated .90 in Experiment $3 \mathrm{~A}$ and .96 in Experiment 3B. Correlations were computed by averaging across individual performance in each task at each orientation. The high positive correlations in each experiment suggest little evidence of a tradeoff between speed and accuracy in these tasks. Nonetheless, as we shall see, the error data yielded many fewer reliable differences than did the RT data.

In each experiment, the measures of selective attention and divided attention at each orientation were subjected to ANOVAS. There was no effect of orientation in either experiment [in Experiment 3A, $F(2,18)=.40, M S_{\mathrm{e}}=$ $1,518.12$, n.s.; in Experiment 3B, $F(2,18)=1.09, M S_{\mathrm{e}}=$ $4,087.93$, n.s.]. Both experiments showed an effect of attention task, with selective attention leading to faster RTs than divided attention did [in Experiment $3 \mathrm{~A}, F(1,9)=$ 62.18, $M S_{\mathrm{e}}=5,551.42, p<.001$; in Experiment 3B, $\left.F(1,9)=94.13, M S_{\mathrm{e}}=4,393.09, p<.001\right]$. Both experiments also revealed a trend toward interaction between orientation and attention task, although only in Experiment 3B did this interaction reach statistical significance [in Experiment 3A, $F(2,18)=2.93, M S_{e}=4,843.77$, $p=.07$; in Experiment 3B, $F(2,18)=10.86, M S_{\mathrm{e}}=$ $1,188.92, p<.001]$. As can be seen in Figure 7, the interaction is due to deteriorating performance in selective attention as axes changed orientation from $0^{\circ}$ to $22.5^{\circ}$ to $45^{\circ}$, while performance in divided attention improved.

Further analyses pointed specifically to the effect being tested in these experiments-namely, to a change in selective attention performance (i.e., filtering interference) with orientation of axes. In Experiment $3 \mathrm{~A}$, interference was $72 \mathrm{msec}$ at $0^{\circ}, 131 \mathrm{msec}$ at $22.5^{\circ}$, and $138 \mathrm{msec}$ at $45^{\circ}\left[F(2,18)=5.06, M S_{\mathrm{e}}=2,550.56, p=.01\right]$. In Experiment 3B, interference was $83 \mathrm{msec}$ at $0^{\circ}, 101 \mathrm{msec}$ at $22.5^{\circ}$, and $140 \mathrm{msec}$ at $45^{\circ}\left[F(2,18)=5.35, M S_{e}=\right.$ $8,404.93, p=.01]$. Newman-Keuls post hoc tests $(.05$ criterion level) indicated that, in both experiments, selective attention at the $0^{\circ}$ orientation was significantly better (i.e., faster) than selective attention at either $22.5^{\circ}$ or $45^{\circ}$. In Experiment 3B, the latter orientations also differed from each other, but in Experiment 3A, they did not. The pattern was generally consistent for individual subjects. 
A.

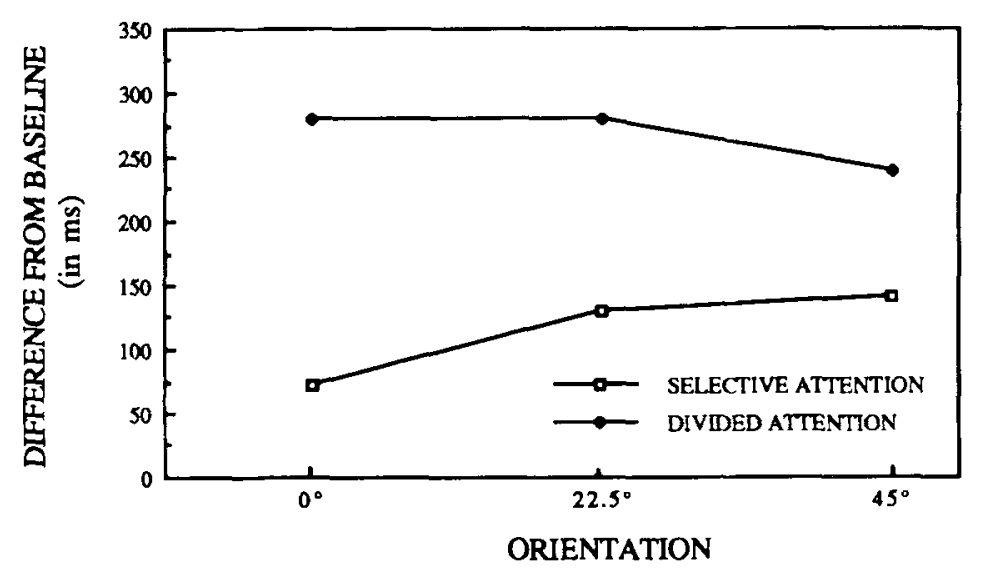

B.

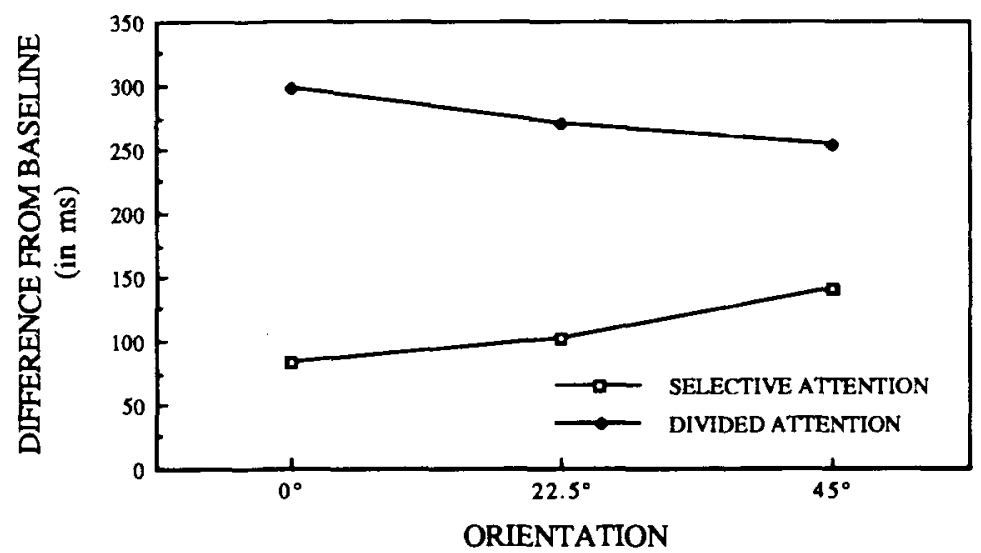

Figure 7. Average reaction time performance in milliseconds in selective attention and divided attention tasks with vibrotactile stimuli created according to three orientations of axes, $0^{\circ}, 22.5^{\circ}, 45^{\circ}$. Testing occurred either with a largely Pacinian range of frequencies (panel $A$; Experiment $3 A$ ) or with a lower range of frequencies (panel B; Experiment 3B).

As is suggested by Figure 7, divided attention performance differed reliably by orientation only in Experiment 3B [in Experiment $3 \mathrm{~A}, F(2,18)=.90, M S_{\mathrm{e}}=$ $6,381.14$, n.s.; in Experiment 3B, $F(2,18)=4.50, M S_{\mathrm{e}}=$ $1,134.78, p=.03$ ]. In Experiment 3B, speed of divided attention dropped from $298 \mathrm{msec}$ at the $0^{\circ}$ orientation to $271 \mathrm{msec}$ at $22.5^{\circ}$ to $254 \mathrm{msec}$ at $45^{\circ}$.

Unfortunately, little of note appeared in the error analyses. The only significant effect was that, in both Experiments $3 \mathrm{~A}$ and $3 \mathrm{~B}$, errors in selective attention were fewer than those in divided attention $\left[F(1,9)=22.75, M S_{\mathrm{e}}=\right.$ $.02, p=.001$, and $F(1,9)=12.51, M S_{\mathrm{e}}=.04, p<$ .01 , respectively].

\section{Discussion}

The principal finding of Experiments $3 \mathrm{~A}$ and $3 \mathrm{~B}$ can be stated easily. Performance at selective attention is affected powerfully by how axes are oriented in vibrotactile perceptual space. The closer the orientation corresponded to the dimensions of pitch and loudness, the better subjects performed in this attention task. These results are opposite what one would expect from early holistic models. The subjects suffered $78 \mathrm{msec}$ of Garner interference on the average when classifying pitch and loudness, indicating again that these dimensions do interact in speeded classification. If Garner interference is explained by holistic processing, itself based in Euclidean distance relations, one should predict that performance would be invariant with how stimuli are oriented in the space. This prediction was clearly disconfirmed. The subjects suffered interference above and beyond the Garner interference at $0^{\circ}$ when the stimulus set was not aligned with the pitch-loudness coordinate axes. The results accord with the view that pitch and loudness are primary dimensions in vibrotactile perceptual space.

These results are particularly important because human beings have little classification experience with vibrotactile dimensions, at least relative to daily encounters at dis- 
tinguishing auditory pitches, loudnesses, and timbres in, say, music appreciation. The results thus indicate greater generality to the results obtained by Melara and Marks (1990a). In three experiments by them, and in two experiments here, we have found that selective attention is best at $0^{\circ}$. The pattern was similar in vibrotaction whether the frequency range was high (Pacinian) or low (Pacinian and non-Pacinian). It is also interesting to note that the psychological differences along dimensions were relatively small in this study (see Craig, 1972; Rothenberg et al., 1977), and appreciably smaller than in Melara and Marks's auditory study. Apparently, the magnitude of stimulus differences is inconsequential to the overall pattern of effects of orientation on RT (cf. Experiment 4 below).

The results suggest the following conclusion: Whatever optional processes appear through experience have little bearing on primacy of dimensions, because uniqueness of orientation is a fundamental characteristic even of primitive dimensions, like those in vibrotaction. Holistic processing of either the mandatory or the optional sort is counterindicated by our findings. Hence, the present results argue against holistic models, including the optional holistic account.

This conclusion is independent of results obtained for performance at divided attention. Nonetheless, results with divided attention were less clear-cut than those with selective attention. In both experiments, divided attention seemed to improve as the axis changed in orientation from $0^{\circ}$ to $45^{\circ}$. This pattern fits with the findings of Melara and Marks (1990a). However, only in Experiment 3B was this effect reliable statistically. This perhaps indicates an effect of experience, as subjects in Experiment 3B had reaped the earlier vibrotactile experiences of Experiment $3 \mathrm{~A}$; in the present study, however, this explanation cannot be evaluated.

In any case, it is important that in both experiments selective and divided attention tended to converge as axes changed orientation toward $45^{\circ}$. A primary dimensions explanation of this convergent pattern, proposed by Melara and Marks (1990a), is that, at $45^{\circ}$, subjects can perform condensation by focusing on only a single primary dimension (e.g., pitch). They respond with one key if a middle value appears, and they respond with the other key if an extreme value appears. According to this explanation, condensation performance is worse at $0^{\circ}$ than at $45^{\circ}$ because subjects must attend to two primary dimensions at the former orientation, but require only one primary dimension at the latter orientation. This coupled with the effects of primary dimensions on selective attention leads to the pattern of convergence (see Figure 7). Results obtained with both auditory dimensions and vibrotactile dimensions are consistent with this explanation.

\section{EXPERIMENTS 4A AND 4B}

Holistic processing is typically conceptualized as a Euclidean map, as we have described in this article. An alternative conceptualization exists, however, in which holistic refers to neither Euclidean nor city-block space, but to unidimensional space. According to this view, the holistic claim that perceivers cannot distinguish a change in one dimension (e.g., pitch) from a change in a second dimension (e.g., loudness) is identical to the claim that stimuli vary on just a single dimension of perceptual experience. Thus, a holistic representation is actually a unidimensional representation.

Experiment 3 demonstrated that subjects were adroit at recognizing changes along the vibrotactile dimensions of pitch and loudness. This was shown by the efficient filtering classifications on these dimensions relative to other dimensions in the space. Advocates of the optional holistic account have claimed, however, that the probability of holistic processing between interacting dimensions is reduced when stimulus differences are relatively large (see, e.g., Foard \& Kemler Nelson, 1984). Thus, the fact that subjects were able to attain such high accuracy in the speeded task could itself have discouraged holistic processing, even though stimulus differences were much smaller in Experiments 3A and 3B than in Melara and Marks's (1990a) experiments with auditory dimensions. The implication is that evidence of holistic processing between vibrotactile dimensions may be evident only for extremely small changes in pitch and loudness, when overall accuracy is relatively low. In other words, when the stimulus differences are tiny, perceivers should be poor at distinguishing a change in pitch from a change in loudness.

In Experiments 4A and 4B, we evaluated this claim by introducing a new unspeeded discrimination paradigm. We reduced stimulus differences until the subjects could no longer discriminate change from no change with $100 \%$ accuracy. We then compared the subjects' ability to identify change with their ability to identify the kind of change. Pairs of vibrotactile stimuli were presented, and they were identical or different in either pitch or loudness. The stimuli in Experiment 4A were higher in frequency than the stimuli in Experiment $4 B$, and thus analogous to the stimuli in Experiments 3A and 3B. The subjects' task was first to identify whether each pair was same or different, and then, if different, to identify whether the change occurred in pitch or in loudness. According to a unidimensional interpretation of holistic processing, even when subjects recognize small changes correctly they should be poor at identifying the specific dimensions of change. According to a primary dimensions account, perceivers process stimuli through a set of specific dimensions; thus subjects' ability to identify the dimensions of change should be good relative to their ability to recognize change alone, even though stimulus differences are small. Experiment 4 therefore permitted us to test a final variant of the early holistic account.

\section{Method}

Subjects. The 10 subjects from Experiments $3 A$ and $3 B$ also participated in Experiments $4 \mathrm{~A}$ and $4 \mathrm{~B}$.

Stimuli, Apparatus, and Procedure. The apparatus was identical to that in the previous experiments. In each experiment, three stimuli were tested, a reference stimulus and two comparison stimuli; one comparison differed from the referent in pitch, the other in loudness. In Experiment 4A, the reference stimulus was $263 \mathrm{~Hz}$ and 
$103 \mathrm{~dB}$; the comparison stimulus that differed in pitch was $276 \mathrm{~Hz}$ and $103 \mathrm{~dB}$; and the comparison stimulus that differed in loudness was $263 \mathrm{~Hz}$ and $106 \mathrm{~dB}$. In Experiment $4 \mathrm{~B}$, the referent was $133 \mathrm{~Hz}$ and $111 \mathrm{~dB}$; a comparison stimulus of $148 \mathrm{~Hz}$ and $114 \mathrm{~dB}$ differed in pitch alone; loudness change was given by a stimulus of $133 \mathrm{~Hz}$ and $117 \mathrm{~dB}$.

On each trial, two signals were presented sequentially for $1,100 \mathrm{msec}$, separated by $500 \mathrm{msec}$. On same trials, the two signals were two presentations of the reference stimulus. On different trials, the reference stimulus was paired with one of the two comparison stimuli. The subjects were instructed to decide whether the two stimuli were the same or different, making their responses on the computer keyboard. Whenever subjects responded "different," they were then asked by computer message to identify the kind of difference-that is, change in pitch or change in loudness. Error feedback was supplied after each trial.

Each subject participated in two blocks of 300 trials, with each block separated by approximately 1 week. Half of the trials in each block were same trials; half of the different trials consisted of a pitch change, and half consisted of a loudness change. The trials were ordered randomly. Each block was performed in approximately $1 \mathrm{~h}$. The two experiments were separated by approximately 2 months.

\section{Results}

At least two approaches exist for comparing subjects' ability in these experiments to detect change with their ability to detect the kind of change. One approach is to examine how often subjects identified the kind of change correctly, given that a change was noticed. In this analysis, subjects' ability to identify stimulus differences is given by the conditional probability

$$
P\left(\mathrm{I}_{\mathrm{c}} \mid \mathrm{A}_{\mathrm{c}}\right)=\frac{P\left(\mathrm{I}_{\mathrm{c}} \cap \mathrm{A}_{\mathrm{c}}\right)}{P\left(\mathrm{~A}_{\mathrm{c}}\right)}
$$

where $P\left(\mathrm{I}_{\mathrm{c}}\right)$ is the probability of identifying a change and $P\left(\mathrm{~A}_{c}\right)$ is the probability that a change actually occurred. $P\left(I_{c} \mid A_{c}\right)$ was .57 in Experiment $4 A$ and .72 in Experiment $4 B$. These values can be examined in the context of subjects' success in identifying changes correctly when they were noticed:

$$
P\left(\mathrm{I}_{\mathbf{k}} \mid \mathrm{A}_{\mathrm{k}}\right)=\frac{P\left(\mathrm{I}_{\mathrm{k}} \cap \mathrm{A}_{\mathrm{k}}\right)}{P\left(\mathrm{~A}_{\mathrm{k}}\right)}
$$

where $P\left(\mathrm{I}_{\mathbf{k}}\right)$ is the probability of identifying a particular kind of change and $P\left(\mathrm{~A}_{\mathrm{k}}\right)$ is the probability that a difference was noticed when that kind of change actually occurred. Averaging across pitch and loudness changes, $P\left(I_{k} \mid A_{k}\right)$ was .78 in Experiment $4 A$ and .96 in Experiment 4B.

Consider the results of Experiment 4A. The subjects in this experiment were at chance level in their ability to determine sameness or difference accurately [i.e., in a one-group $t$ test with $\mathrm{H}_{0}: \mu=.5, t(9)=1.43$, n.s.]. Nonetheless, when a change was noticed, the subjects were very accurate at determining whether that change occurred in pitch or in loudness. A similar pattern was obtained in Experiment 4B. A full summary of detection probabilities appears in Table 1.

A second approach, one that permits us to compare statistically the ability to identify change with the ability to identify the kind of change, is to perform a signal detec-
Table 1

Summary of Detection Performance in an Unspeeded Same-Different Task in Which Pairs of Vibrotactile Stimuli Were Identical or Different in Either Pitch or Loudness (Experiments $4 \mathrm{~A}$ and $\mathbf{4 B}$ )

\begin{tabular}{lcc}
\hline \multicolumn{1}{c}{ Detection Probability } & Experiment 4A & Experiment 4B \\
\hline$P$ ("different"| different) & .57 & .72 \\
$P$ ("same"| same) & .69 & .86 \\
$P$ ("different pitch" $\left.\mid A_{\mathbf{k}(p)}\right)$ & .80 & .94 \\
$P$ ("different loudness" $\left.\mid A_{\mathbf{k}(1)}\right)$ & .77 & .98 \\
$P$ ("different pitch" $\mid$ same) & .21 & .12 \\
$P$ ("different loudness"| same) & .21 & .03 \\
\hline
\end{tabular}

Note-P ("different" | different) is the probability that subjects responded "different" given that two stimuli actually differed; $P\left(A_{k(p)}\right)$ is the probability that a difference in pitch was noticed when that kind of change actually occurred (see text for details).

tion analysis. We compared two $d^{\prime}$ measures: sensitivity to detect change, and sensitivity to detect kind of change. Because each analysis involved a same-different decision between only two stimuli (i.e., reference stimulus and comparison stimulus), which is what Macmillan and Creelman (1991, p. 155) call the "reminder" experiment, sensitivity was computed by using the $d^{\prime}$ equation for the basic yes/no design:

$$
d^{\prime}=z(\mathrm{H})-z(\mathrm{FA}),
$$

where $z(\mathrm{H})$ is the $z$-transformed hit rate and $z(\mathrm{FA})$ is the $z$-transformed false alarm rate. For sensitivity to detect change, hits were defined as the proportion of correctly identified differences, and false alarms as the proportion of same trials called "different." Analogously, for sensitivity to detect kind of change, hits were defined as the proportion of trials in which a difference was correctly identified by kind; false alarms were the proportion of same trials in which a particular kind of difference was identified. Sensitivity was derived separately for each subject and then averaged. Table 2 summarizes averaged $d^{\prime}$ and the variance in $d^{\prime}$ for each of three conditions-detecting change, detecting pitch change, and detecting loudness change-in Experiments 4A and 4B.

All $d$ 's were statistically greater than zero. On the average, the subjects' sensitivity in Experiment $4 B$ was superior to their sensitivity in Experiment 4A. In Experiment $4 \mathrm{~A}$, the subjects were better at detecting a change in pitch (1.30) than they were at detecting a change in loudness (.61). The average $d^{\prime}$ for detecting kind of change (.95) was significantly greater than the average $d^{\prime}$ for detecting change alone (.68). In Experiment 4B, the subjects' ability to detect loudness changes (3.03) exceeded their ability to detect pitch changes (1.37). Again, critically, the average $d^{\prime}$ for detecting kind of change (2.20) was actually greater (in a statistical sense) than $d^{\prime}$ for change alone (1.74). The substantive point is that the subjects' overall performance at detecting kind of change was no worse than their performance at detecting change alone.

We also examined two measures of decision bias. For change alone, pitch change, and loudness change, we derived $c$, the criterion measure, and $\mathcal{L}^{\prime}$, the value of the 
Table 2

Summary of Signal Detection Analyses in an Unspeeded Same-Different Task in Which Pairs of Vibrotactile Stimuli Were Identical or Different in Either Pitch or Loudness (Experiments $\mathbf{A A}$ and $\mathbf{4 B}$ )

\begin{tabular}{lccccc}
\hline \multicolumn{1}{c}{ Conditions } & \multicolumn{1}{c}{$d^{\prime}$} & $\operatorname{var}\left(d^{\prime}\right)$ & $c$ & $\operatorname{var}(c)$ & $L$ \\
\hline \multicolumn{7}{c}{ Experiment 4A } \\
Change alone & 0.6807 & .0065 & .1636 & .0016 & 1.2922 \\
Kind change: pitch & 1.2958 & .0186 & .4713 & .0047 & 4.0803 \\
Kind change: loudness & 0.6087 & .0180 & .6172 & .0045 & 2.3751 \\
\multicolumn{7}{c}{ Experiment 4B } \\
Change alone & 1.7438 & .0086 & .2258 & .0022 & 2.4758 \\
Kind change: pitch & 1.3725 & .0193 & .5099 & .0048 & 5.0099 \\
Kind change: loudness & 3.0263 & .0355 & .4083 & .0089 & 17.2045 \\
\hline
\end{tabular}

Note- $\operatorname{var}\left(d^{\prime}\right)$ is the variance of the sensitivity measure, $d^{\prime} ; \operatorname{var}(c)$ is the variance of the bias measure, $c$.

likelihood ratio at $c$ (see Macmillan \& Creelman, 1991). These measures are summarized in Table 2 . A positive value of $c$ or, alternatively, a value of $\mathcal{L}$ greater than 1.0 , means that subjects were biased toward responding "same." One can see in Figure 2 that a same bias was present in each of the conditions; all criterion measures were significantly greater than zero. Moreover, subjects showed more bias for decisions about kind of change than for decisions about change alone. In other words, subjects had a greater tendency to commit misses for questions of kind than for questions of simple change.

\section{Discussion}

Experiments $4 \mathrm{~A}$ and $4 \mathrm{~B}$ yielded patent evidence that perceivers of vibrotactile stimuli are no worse at detecting the kind of change than they are at detecting change alone, despite extremely small stimulus differences. Indeed, we found that even when subjects were very poor at discriminating change from absence of change, their decisions about the kind of change were relatively accurate. In other words, subjects rarely mistook a change in pitch for a change in loudness once they had noticed that a change had occurred. Similarly, signal detection analyses indicated that subjects' sensitivity to the kind of change was at least as good as their sensitivity to change alone. There was little evidence to suggest that these stimuli were perceived holistically. Thus, Experiments $4 \mathrm{~A}$ and 4B serve as additional evidence against the currently popular holistic processing models.

The subjects displayed considerable decision bias in these experiments. At present, we cannot speculate on the causes of the bias, nor can we determine the nature of the decision strategy (e.g., independent observations, differencing, etc.) that led to these response effects. At best, we may conclude that the subjects probably utilized multiple criteria in these experiments, a different one for each decision that they reached. We emphasize, though, that the issue of decision rules is independent of the perceptual issue under study - namely, that of whether subjects detect vibrotactile change without experiencing the kind of change. With respect to the latter issue, we may conclude that the evidence is negative. The fact that clear decisional differences are obtained in the absence of any perceptual differences only reinforces this conclusion.

\section{GENERAL DISCUSSION}

Four full experiments support our claim that perceivers process multidimensional stimuli through a set of primary dimensions, even when the stimuli are formed from dimensions that interact. Experiment 1 confirmed that vibrotactile pitch and loudness do interact in speeded classification. Specifically, subjects were unable to attend to one of these dimensions, suffering Garner interference from orthogonal variation of the other dimension. Experiment 2 demonstrated that Garner interference does not diminish even after extensive practice at the classification task. Vibrotactile interactions thus fit our operational definition of a mandatory process. Experiment 3 showed that pitch and loudness are the psychologically important dimensions of vibrotactile perceptual space. Stimuli are apparently interpreted in terms of pitch and loudness even when constructed according to axes at other orientations in the space. Finally, in Experiment 4, subjects' ability to specify changes either in pitch or in loudness was shown to be excellent despite extremely small stimulus changes. Experiment 4 thereby negated a central prediction of a modified holistic model. Collectively, the various results of this study are persuasive in disconfirming the most ubiquitous holistic accounts. The experiments also suggest several new ideas about how best to conceptualize (1) the difference between interacting dimensions and separable dimensions and (2) the nature of perceptual interaction. We will discuss these ideas next.

\section{The Relation Between Interacting and Separable Dimensions}

By demonstrating that vibrotactile pitch and loudness interact, but are also primary dimensions, the current research discredits a notion long assumed to distinguish interacting dimensions from separable dimensions. This is the claim that specific dimensions of experience are critical only when Garner interference is absent in classification. The mere presence of Garner interference was believed to 
indicate that perceivers process stimulus wholes and consider constituent dimensions to be unimportant. The present experiments disconfirm this idea. Whether the stimulus dimensions are interacting or separable, and whether the task consists of classification or discrimination, perceivers are aware of and actively use the features of multidimensional stimuli. We call these features the primary dimensions of the stimulus, because such features appear to enjoy primacy in perception. ${ }^{1}$

One way to formulate this new conception is to consider performance in speeded classification to be some function of the spatial orientation of axes. Consider, for example, a power function:

$$
D_{\alpha}=\alpha^{n}+g,
$$

$D_{\alpha} \geq 0, n>0, g \geq 0,-45<\alpha<45$, where $D$ refers to perceptual distances among stimuli within a category (e.g., distance between $A$ and $B$ in Figure 2), $\alpha$ refers to the orientation of stimulus axes in perceptual space, and $g$ refers to the effects on classification of varying primary dimensions orthogonally. As axes are oriented progressively farther from the unique orientation of the space, stimuli from the same category (i.e., assigned to the same response) are displaced to a greater extent along the primary dimension of classification. Intracategory distance (or intracategory variability) undermines classification efficiency (see Figure 2). Accordingly, the greater $D_{\alpha}$, the worse is performance in speeded classification.

To determine the orientation of the axes corresponding to the primary dimensions of the stimulus, we derive the minimum of the classification function. That is, we take the derivative of the function with respect to $\alpha$,

$$
\frac{\partial D_{\alpha}}{\partial \alpha}=n \alpha^{n-1},
$$

set it to zero,

$$
\frac{\partial D_{\alpha}}{\partial \alpha}=0
$$

and solve for $\alpha$,

$$
\begin{aligned}
n \alpha^{n-1} & =0 \\
\alpha & =0 .
\end{aligned}
$$

A power function where $\alpha=0$ (i.e., $0^{\circ}$ orientation) and $n=2$ is consistent with the results of the present study. Note that the magnitude of Garner effects, $g$, is the value of $D \alpha$ at its minimum.

According to the conception outlined here, both separable dimensions and interacting dimensions obey this function. The only difference between these two types of dimensions lies in the value of the Garner constant, $g$. With separable dimensions, $g=0$; there is no Garner interference. Thus, $\left.D_{\alpha} \mid\left[\partial D_{\alpha} / \partial_{\alpha}\right)=0\right]=0$ for both filtering tasks and baseline tasks. Classification of separable dimensions entails no intracategory distance. Subjects attend only to one stimulus value within each category, successfully ignoring the irrelevant dimension. With interacting dimensions, on the other hand, $g>0$; there is a measurable degree of Garner interference. Hence, $D_{\alpha} \mid\left[\left(\partial D_{\alpha} / \partial_{\alpha}\right)=\right.$ $0]>0$. In other words, even at its minimum, the performance function yields a value indicative of inefficiency. The notion that only separable dimensions enjoy a primary orientation is incorrect; a primary orientation may inhere in any perceptual space. Rather, in this view, what characterizes separability as distinct from interaction is that only with separable pairs of dimensions does $D_{\alpha}$ equal zero at the primary orientation.

The idea that $g$ exceeds zero when dimensions interact merely restates the empirical result. More is implied by the assertion that $D_{\alpha}$ exceeds zero when dimensions interact; this suggests that each category in a filtering task contains more than one perceptual value. Still, these ideas are poorly articulated. What is needed is a clearer understanding of what mechanisms underlie dimensional interaction, particularly as defined by the presence of Garner interference. In the remainder of this article, we will explore several alternative approaches to this problem.

\section{What Causes Garner Interference?}

In our view, three promising approaches-that is, three useful models-exist for answering this question. Each approach provides a slightly different emphasis on the underlying psychological processes; but each concentrates on elucidating the constant $g$ in Equation 6. The approaches by no means exclude one another. Indeed, dimensional interaction may eventually need to be explained by some combination of the proposed processes. Unfortunately, the present set of data leaves us unable to evaluate these approaches. This issue must be left for future study.

The first model was suggested earlier when the failures of early holistic models were discussed: $g$ can be thought to reflect the processing time required to create a cityblock map. According to this model, a unidimensional representation suffices for baseline classification, at least for stimulus sets based in the primary orientation. Perceivers must derive a multidimensional representation, however, when classifying stimuli in a filtering task. The idea, then, is that achieving the multidimensional representation is more difficult with interacting dimensions than with separable dimensions. The time required corresponds to the amount of Garner interference at the primary orientation, $g$. Further amounts of interference, from sets based in nonprimary orientations, are attributable to $\alpha^{n}$, which itself corresponds to distance relations in city-block space (see Figure 2). City-block maps of separable dimensions are presumed to incur no measurable processing lag: $g=0$. Because of the city-block distance function, however, $\alpha^{n}$ always exceeds zero at nonprimary orientations, whether dimensions are separable or interacting.

By attributing Garner interference to the formation of psychophysical maps, this map-making model credits interference effects at primary and nonprimary orientations to different aspects of the spatial representation. Another explanation of dimensional interaction, suggested by Melara and Marks (1990a), states that a value on one dimension 
can alter how the perceiver experiences a value along another dimension. The experience of high intensity in vibrotactile perception may change between two stimuli that differ even slightly in frequency. To be sure, we selected values of loudness and pitch in our experiments on the basis of psychophysical considerations. Nevertheless, one might entertain the possibility that, when dimensions interact, varying an irrelevant dimension causes a perceptual change on the relevant dimension. Within any one loudness category, for example, there could fall two projections on the loudness axis-namely, a high-pitched loud value and a low-pitched loud value. Of course, in line with our results, within-category projections would be closest for categories at the primary orientation.

This contextual model of dimensional interaction focuses on $D_{\alpha}$ in Equation 6, making interference at all orientations the result of a displacement of values along primary dimensions. Still, one must distinguish contextual changes at the primary orientation, $g$, from those at nonprimary orientations, $\alpha^{n}$, because in this view only the changes wrought through $g$ are de facto perceptual. In this respect, the contextual model provides a novel interpretation of dimensional interaction; certain primary dimensions can serve as the lens through which to perceive other primary dimensions. Further details of the contextual model are provided by Melara and Marks (1990a).

The third model takes a neoholistic approach. The idea is that change occurring along one dimension is more or less similar phenomenally to change occurring along another dimension. In other words, the two dimensions within any pair are, to some degree, confusable. The model, then, interprets $g$ as the degree of dimensional similarity between two psychological continua. This view has intuitive appeal; anyone who has taught a perception course has probably experienced frustration in describing to students what constitutes a change in color saturation versus a change in lightness. Changes occurring along these two dimensions are certainly more similar phenomenally than changes occurring along, say, hue and shape. In general, it seems obvious that perceptual dimensions do vary in how similar they are to one another.

The results of the present study even jibe with the concept of dimensional similarity. In Experiment 4, for example, we showed that subjects were highly accurate at distinguishing changes in pitch from changes in loudness. Nonetheless, the subjects were not perfect; they sometimes mistook a change in physical frequency for a change in psychological loudness. Presumably, certain other pairs of dimensions would be less likely to lead to such confusions.

A model of dimensional similarity bears a certain resemblance to the early holistic models evaluated, and rejected, in this article. Both ascribe Garner interference to the failure to identify stimulus changes precisely. These approaches differ, however, because holistic implies that two dimensions are indistinguishable and hence unidimensional, whereas the model proposed here is intrinsically multidimensional. Dimensions can have greater or lesser similarity without ever being unitary. Indeed, our view is that two dimensions must be psychologically separate and individually important-that is, primary-in order to interact in the first place (see Melara \& Marks, 1990a). From this, the possibility follows naturally that dimensions may be both similar and primary.

The construct of dimensional similarity permits an interesting way to characterize information integration. Human beings have a predilection to integrate different sources of information. Our phenomenal impression of the world is dominated by wholes; we experience perfumes, faces, melodies, and pin pricks. The need of human beings to integrate multiple dimensions was recognized long ago by the Gestalt psychologists. A new interpretation is that dimensional similarity biases perceivers toward integration, with information from interacting dimensions being integrated more readily than information from separable dimensions. In this view, Garner interference might itself reflect a mandatory tendency by perceptual systems to blend separate sources of information.

But dimensional interaction is not the same as perceptual fusion; even two very similar dimensions are not a blob. Thus, the tendency to integrate information need not be characterized in terms of early holistic processes. Indeed, the present research suggests that integration is the successor rather than the predecessor of dimensional processing. For we have shown that even dimensions as similar phenomenally and as jejune experientially as vibrotactile pitch and loudness enjoy a primacy in perception.

\section{REFERENCES}

BéKÉSY, G. von (1959). Similarities between hearing and skin sensations. Psychological Review, 66, 1-22.

Békésy, G. voN (1967). Sensory inhibition. Princeton, NJ: Princeton University Press.

Beranek, L. L. (1988). Levels, decibels, and spectra. In Noise and vibration control (rev. ed, pp. 25-44). Washington, DC: Institute of Noise Control Engineering.

Craig, J. C. (1972). Difference threshold for intensity of tactile stimuli. Perception \& Psychophysics, 11, 150-152.

EGETH, H. (1967). Selective attention. Psychological Bulletin, 67, 41-57.

FitTs, P. M., Biederman, I. (1965). S-R compatibility and information reduction. Journal of Experimental Psychology, 69, 408-412.

FonRd, C. F., Kemler Nelson, D. G. (1984). Holistic and analytic modes of processing: The multiple determinants of perceptual analysis. Journal of Experimental Psychology: General, 113, 94-111.

FreemAN, A. W., Johnson, K. O. (1982). A model accounting for effects of vibratory amplitude on responses of cutaneous mechanoreceptors in macaque monkey. Joumal of Physiology, 323, 43-64.

GARNER, W. R. (1974a). Attention: The processing of multiple sources of information. In E. C. Carterette \& M. P. Friedman (Eds.), Handbook of perception (Vol. 2, pp. 23-59). New York: Academic Press.

GARNER, W. R. (1974b). The processing of information and structure. Potomac, MD: Erlbaum.

GARNER, W. R., Felfoldy, G. L. (1970). Integrality of stimulus dimensions in various types of information processing. Cognitive Psychology, 1, 225-241.

Gescheider, G. A. (1976). Evidence in support of the duplex theory of mechanoreception. Sensory Processes, 1, 68-76.

Gescheider, G. A., Frisina, R. D., \& Verrillo, R. T. (1979). Selective adaptation of vibrotactile thresholds. Sensory Processes, 3, 37-48. Grau, J. W., \& Kemler Nelson, D. G. (1988). The distinction be- 
tween integral and separable dimensions: Evidence for the integrality of pitch and loudness. Journal of Experimental Psychology: General, 117, 347-370.

IGGo, A. (1985). Sensory receptors in the skin of mammals and their sensory functions. Revue Neurologique, 141, 599-613.

IMAI, S., GARNER, W. R. (1965). Discriminability and preference for attributes in free and constrained classification. Journal of $\mathbf{E x}$ perimental Psychology, 69, 596-608.

KEMLER, D. G. (1983a). Exploring and reexploring issues of integrality, perceptual sensitivity, and dimensional salience. Journal of Experimental Child Psychology, 36, 365-379.

KemLER, D. G. (1983b). Holistic and analytic modes in perceptual and cognitive development. In T. Tighe \& B. E. Shepp (Eds.), Perception, cognition, and development: Interactional analyses (pp. 77-102). Hillsdale, NJ: Erlbaum.

Khanna, S., \& Sherrick, C. E. (1981). The comparative sensitivity of selected receptor systems. In T. Gualtierotti (Ed.), Symposium on vestibular function and morphology (pp. 337-348). New York: Springer-Verlag.

KöHLER, W. (1940). Dynamics in psychology. New York: Liveright.

Lockhead, G. R. (1972). Processing dimensional stimuli: A note. Psychological Review, 79, 410-419.

LOCKHEAD, G. R. (1979). Holistic versus analytic process models: A reply. Joumal of Experimental Psychology: Human Perception \& Performance, 5, 746-755.

Macmillan, N. A., Creelman, C. D. (1991). Detection theory: A user's guide. Cambridge: Cambridge University Press.

Melara, R. D. (1990, November). Mandatory processes: Consider your options. Paper presented at the 31st Annual Meeting of the Psychonomic Society, New Orleans.

Melara, R. D., Marks, L. E. (1990a). Perceptual primacy of dimensions: Support for a model of dimensional interaction. Joumal of Experimental Psychology: Human Perception \& Performance, 16, 398-414.

Melara, R. D., Marks, L. E. (1990b). Processes underlying dimensional interactions: Correspondences between linguistic and nonlinguistic dimensions. Memory \& Cognition, 18, 477-495.

Pomerantz, J. R. (1983). Global and local precedence: Selective attention in form and motion perception. Journal of Experimental Psychology: General, 112, 515-540.

Pomerantz, J. R. (1986). Visual form perception: An overview. In E. C. Schwab \& H. C. Nusbaum (Eds.), Pattern recognition by humans and machines: Visual perception (pp. 1-30). New York: Academic Press.

Rothengerg, M., Verrillo, R. T., Zahorian, S. A., Brachman, M. L., BolanowsKr, S. J., JR. (1977). Vibrotactile frequency for encoding a speech parameter. Journal of the Acoustical Society of America, 62, 1003-1012.

SHEPP, B. E. (1989). On perceiving objects: Holistic versus featural properties. In B. E. Shepp \& S. Ballesteros (Eds.), Object perception: Structure and process (pp. 203-233). Hillsdale, NJ: Erlbaum.
Sherrick. C. E., Cholewiak, R. W. (1986). Cutaneous sensitivity. In K. R. Boff, L. Kaufman, \& J. P. Thomas (Eds.), Handbook of perception and human performance (Vol. 1, 12-1-12-58). New York: Wiley.

Smith, L. B., \& KemLeR, D. G. (1977). Developmental trends in free classification: Evidence for a new conceptualization of perceptual development. Journal of Experimental Child Psychology, 24, 279-298.

SMITH, L. B., KEMLER, D. G. (1978). Levels of experienced dimensionality in children and adults. Cognitive Psychology, 10, 502-532.

Smith, J. D., \& Kemler Nelson, D. G. (1984). Overall similarity in adults' classification: The child in all of us. Journal of Experimental Psychology: General, 113, 137-159.

TAYLOR, B. (1977). Dimensional interactions in vibrotactile information processing. Perception \& Psychophysics, 21, 477-481

VERRILLO, R. T. (1963). Effect of contactor area on the vibrotactile threshold. Joumal of the Acoustical Society of America, 35, 1962-1966.

VERRILL, R. T. (1966). Vibrotactile sensitivity and the frequency response of the Pacinian corpuscle. Psychonomic Science, 4, 135-136.

VERRILLO, R. T. (1985). Psychophysics of vibrotactile stimulation. Journal of the Acoustical Society of America, 77, 225-232.

WARD, T. B. (1983). Response tempo and separable-integral responding: Evidence for an integral-to-separable processing sequence in visual perception. Journal of Experimental Psychology: Human Perception \& Performance, 9, 103-112.

Wertheimer, M. (1958). Principles of perceptual organization (abridged translation by $\mathbf{M}$. Wertheimer). In D. S. Beardslee \& $M$. Wertheimer (Eds.), Readings in perception (pp. 115-137). Princeton, NJ: Van Nostrand-Reinhold. (Original work published in Psychologische Forschung, 41 [1923], 301-350)

WINER, B. J. (1971). Statistical principles in experimental design. New York: McGraw-Hill.

WooD, C. C. (1975). Auditory and phonetic levels of processing in speech perception: Neurophysiological and information-processing analyses. Journal of Experimental Psychology: Human Perception \& Performance, 1, 3-20.

WrIGHT, R. D. (1986). Amiga 1000 hardware timing and reaction-time key interfacing. Behavior Research Methods, Instruments, \& Computers, 18, 463-465.

\section{NOTE}

1. We stress that our analyses and conclusions concern only interactions between perceptual (i.e., psychologically real) dimensions, such as pitch and loudness, not between physical dimensions, such as frequency and intensity. The issue of physical interactions (e.g., color metamerism) is separate and is not addressed by this research (see Melara \& Marks, 1990a, for further discussion of this issue).

(Manuscript received August 20, 1991; revision accepted for publication January $7,1992$. 